\title{
Pan-Pseudothrombocytopenia in COVID-19: A Harbinger for Lethal Arterial Thrombosis?
}

\author{
Patrick KuhIman, MD; Julio Nasim, MD; and Michael Goodman, MD
}

Pseudothrombocytopenia in the setting of COVID-19-associated coagulopathy prompts the question whether it is representative of increased platelet aggregation activity in vivo.

\section{Patrick Kuhlman is a PGY-6 Resident, and Michael Goodman is an Assistant Professor, both in the Hematology- Oncology Fellowship Program; Julio Nasim is a PGY-5 Resident in the Infectious Disease Fellowship Program; all at Wake Forest University School of Medicine in Salem, North Carolina, and the W.G. (Bill) Hefner VA Medical Center in Salisbury, North Carolina. Correspondence: Patrick Kuhlman} (pkuhlman@wakehealth.edu)

Fed Pract. 2020;37(8):354-358. doi:10.12788/fp.0032 n late 2019 a new pandemic started in Wuhan, China, caused by the severe acute respiratory syndrome coronavirus 2 (SARS-CoV-2) due to its similarities with the virus responsible for the SARS outbreak of 2003. The disease manifestations are named coronavirus disease 2019 (COVID-19). ${ }^{1}$

Pseudothrombocytopenia, or platelet clumping, visualized on the peripheral blood smear, is a common cause for artificial thrombocytopenia laboratory reporting and is frequently attributed to laboratory artifact. In this case presentation, a critically ill patient with COVID-19 developed panpseudothrombocytopenia (ethylenediaminetetraacetic acid [EDTA], sodium citrate, and heparin tubes) just prior to his death from a ST-segment elevation myocardial infarction (STEMI) in the setting of therapeutic anticoagulation during a prolonged hospitalization. This case raises the possibility that pseudothrombocytopenia in the setting of COVID19 critical illness may represent an ominous feature of COVID-19-associated coagulopathy $(\mathrm{CAC})$. Furthermore, it prompts the question whether pseudothrombocytopenia

TABLE Hospitalization Laboratory Values and Therapeutics

\begin{tabular}{|c|c|c|c|c|c|c|c|c|c|}
\hline \multirow[b]{2}{*}{ Variables } & \multicolumn{9}{|c|}{ Hospital Day } \\
\hline & 1 & 2 & 3 & 4 & 5 & 6 & 7 & 8 & 9 \\
\hline Max patient temperature, $\mathrm{C}$ & 38.4 & 38.8 & 38.0 & 38.3 & 38.8 & 39.5 & 39.1 & 38.7 & 39.2 \\
\hline \multicolumn{10}{|l|}{ Medications } \\
\hline Anticoagulants & \multicolumn{6}{|c|}{ Heparin } & \multicolumn{3}{|c|}{ Argatroban } \\
\hline \multirow[t]{3}{*}{ Antibiotics } & \multicolumn{8}{|c|}{ Ceftriaxone } & Linezo \\
\hline & \multicolumn{8}{|c|}{ Doxycycline } & \\
\hline & \multicolumn{4}{|c|}{ Hydroxychloroquine } & & & & & \\
\hline Vasopressors & & Norepinephrine & & & & & Norepinephrine & & \\
\hline \multicolumn{10}{|l|}{ Laboratory Tests } \\
\hline \multicolumn{10}{|l|}{ Partial thromboplastin time, $\mathrm{s}$} \\
\hline $\min$ & -- & 99.2 & 47.1 & -- & 41.2 & 51 & 46 & 41.4 & 34.6 \\
\hline $\max$ & 52.1 & $>180$ & 103.7 & 81.7 & 59.4 & 81.5 & 57.8 & 68.7 & 74.8 \\
\hline D-dimer, ng/mL & $>20$ & -- & -- & $>20$ & $>20$ & -- & -- & -- & -- \\
\hline Ferritin, $\mathrm{mcg} / \mathrm{mL}$ & -- & -- & 2,603 & -- & -- & -- & -- & -- & -- \\
\hline Lactic acid dehydrogenase, U/L & -- & -- & 1,188 & -- & -- & -- & -- & -- & -- \\
\hline White blood cells, $\mathrm{K} / \mu \mathrm{L}$ & 8.90 & 9.56 & 9.62 & 8.63 & 7.99 & 6.70 & 7.89 & 9.55 & 11.00 \\
\hline Lymphocytes, $\mu \mathrm{L}$ & 0.24 & 0.80 & 0.38 & 0.26 & 0.31 & 0.34 & 0.39 & -- & 0.33 \\
\hline Hemoglobin, g/dL & 15.3 & 14.0 & 11.5 & 11.1 & 11.1 & 8.9 & 8.6 & 8.8 & 8.3 \\
\hline Platelet count, $\mathrm{K} / \mu \mathrm{L}$ & 165 & 137 & 168 & 181 & -- & 191 & 187 & 200 & 230 \\
\hline
\end{tabular}


in this setting is representative of increased platelet aggregation activity in vivo.

\section{CASE PRESENTATION}

A 50-year-old African American man who was diagnosed with COVID-19 3 days prior to admission presented to the emergency department of the W.G. (Bill) Hefner VA Medical Center in Salisbury, North Carolina, with worsening dyspnea and fever. His primary chronic medical problems included obesity (body mass index, 33), type 2 diabetes mellitus (hemoglobin $\mathrm{A}_{1 \mathrm{c}} 2$ months prior of $6.6 \%$ ), migraine headaches, and obstructive sleep apnea. Shortly after presentation, his respiratory status declined, requiring intubation. He was admitted to the medical intensive care unit for further management.

Notable findings at admission included $>20 \mathrm{mcg} / \mathrm{mL}$ FEU D-dimer (normal range, $0-0.56 \mathrm{mcg} / \mathrm{mL}$ FEU), $20.4 \mathrm{mg} / \mathrm{dL}$ C-reactive protein (normal range, $<1 \mathrm{mg} / \mathrm{dL}$ ), $30 \mathrm{~mm} / \mathrm{h}$ erythrocyte sedimentation rate (normal range, $0-25 \mathrm{~mm} / \mathrm{h}$ ), and $3.56 \mathrm{ng} / \mathrm{mL}$ procalcitonin (normal range, $0.05-1.99 \mathrm{ng} / \mathrm{mL}$ ). Patient's hemoglobin and platelet counts were normal. Empiric antimicrobial therapy was initiated with ceftriaxone (2 g IV daily) and doxycycline (100 mg IV twice daily) due to concern of superimposed infection in the setting of an elevated procalcitonin.

A heparin infusion was initiated (5,000 U IV bolus followed by continuous infusion with goal partial thromboplastin time [PTT] of $1.5 \mathrm{x}$ the upper limit of normal) on admission to treat CAC. Renal function worsened requiring intermittent renal replacement therapy on day 3 . His lactate dehydrogenase was elevated to 1,188 U/L (normal range: 100-240 U/L) and ferritin was elevated to $2,603 \mathrm{ng} / \mathrm{mL}$ (normal range: $25-350 \mathrm{ng} / \mathrm{mL}$ ) (Table). Initial neuromuscular blockade and prone positioning maneuvers were instituted to optimize oxygenation based on the latest literature for respiratory distress in the COVID-19 management. ${ }^{2}$

Intermittent norepinephrine infusion $(5 \mathrm{mcg} / \mathrm{min}$ with a $2 \mathrm{mcg} / \mathrm{min}$ titration every 5 minutes as needed to maintain mean arterial pressure of $>65 \mathrm{~mm} \mathrm{Hg}$ )

\begin{tabular}{|c|c|c|c|c|c|c|c|c|c|c|c|c|c|}
\hline \multicolumn{13}{|c|}{ Hospital Day } & \multirow{2}{*}{$\begin{array}{c}\text { Reference } \\
\text { Range }\end{array}$} \\
\hline 10 & 11 & 12 & 13 & 14 & 15 & 16 & 17 & 18 & 19 & 20 & 21 & 22 & \\
\hline 38.9 & 38.7 & 38.5 & 38.1 & 37.7 & 37.8 & 38.3 & 37.7 & 39.4 & 38.9 & 38.2 & 37.3 & 38.3 & \\
\hline \multicolumn{13}{|c|}{ Argatroban } & \\
\hline & \multicolumn{12}{|c|}{ Cefepime } & \\
\hline & & & & & & & & & & Vancomycin & & & \\
\hline & & & & & & Norepinephrine & & & & & Norepinephrine & & \\
\hline-- & -- & -- & -- & 69.9 & 62.4 & 58.4 & 71.8 & 68 & 128.6 & 89.3 & -- & -- & $24.0-35.6$ \\
\hline 82.4 & 74.6 & 84.1 & 82.3 & 108.1 & 78.5 & $>180$ & 80 & 75.2 & 128.6 & $>180$ & 85.4 & 95.2 & -- \\
\hline 20.00 & 18.48 & 15.72 & 15.16 & -- & -- & 12.50 & -- & -- & -- & -- & 11.96 & -- & $0.00-0.56$ \\
\hline 1,585 & -- & 1,027 & 1,024 & -- & -- & 644 & -- & -- & -- & -- & -- & -- & $25-350$ \\
\hline 626 & 515 & 463 & 477 & -- & -- & 387 & -- & -- & -- & -- & -- & -- & $100-240$ \\
\hline 13.82 & 13.37 & 12.44 & 11.40 & -- & 8.34 & 6.63 & 6.23 & 5.90 & -- & 13.76 & 12.98 & 12.93 & $4.87-9.47$ \\
\hline 0.63 & 0.53 & 1.62 & 0.57 & -- & 0.92 & 0.65 & -- & 0.59 & -- & 0.58 & 0.50 & 0.91 & $13.11-16.01$ \\
\hline 8.6 & 8.5 & 7.9 & 8.9 & -- & 8.5 & 8.2 & 8.0 & 7.9 & -- & 7.5 & 6.9 & 6.5 & $150-400$ \\
\hline 241 & 207 & 177 & 181 & & & & & & telet & Imping & & & \\
\hline
\end{tabular}


FIGURE 1 Blood Smears Showing Platelet Clumping in All 3 Tubes

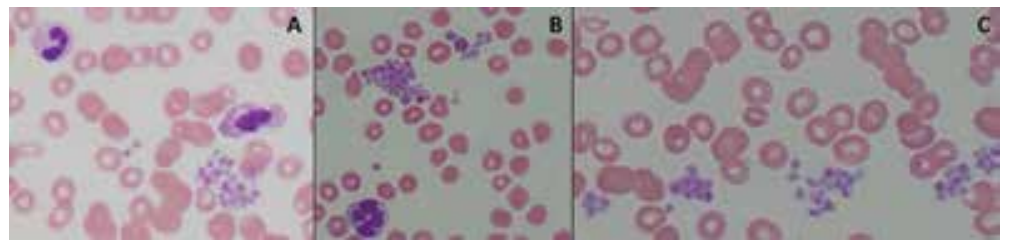

A, ethylenediaminetetraacetic acid; B, citrated; C, heparinized.

\section{FIGURE 2 Electrocardiograms}

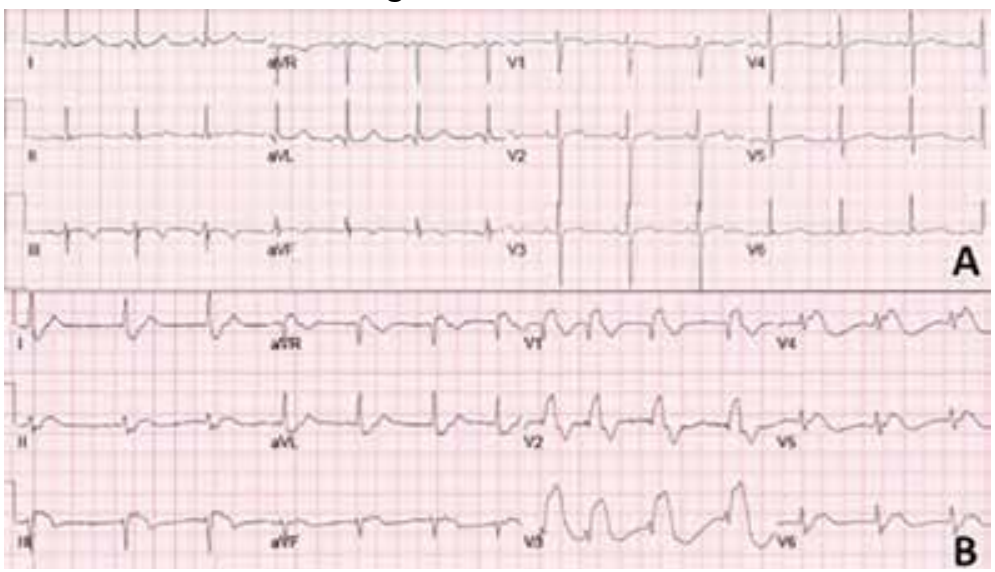

A, Hospital day 5; B, Hospital day 22.

was required for hemodynamic support throughout the patient's course. Several therapies for COVID-19 were considered and were a reflection of the rapidly evolving literature during the care of patients with this disease. The patient originally received hydroxychloroquine ( $200 \mathrm{mg}$ by mouth twice daily) in accordance with the US Department of Veterans Affairs (VA) institutional protocol between day 2 and day 4; however, hydroxychloroquine was stopped due to concerns of QTc prolongation. The patient also received 1 unit of convalescent plasma on day 6 after being enrolled in the expanded access program. ${ }^{3}$ The patient was not a candidate for remdesivir due to his unstable renal function and need for vasopressors. Finally, interleukin-6 inhibitors also were considered; however, the risk of superimposed infection precluded its use.

On day 7 antimicrobial therapy was transitioned to linezolid (600 mg IV twice daily) due to the persistence of fever and a portable chest radiograph revealing diffuse infiltrates throughout the bilateral lungs, worse compared with prior radiograph on day 5 , suggesting a worsening of pneumo- nia. On day 12 , the patient was transitioned to cefepime (1 gram IV daily) to broaden antimicrobial coverage and was continued thereafter. Blood cultures were negative throughout his hospitalization.

Given his worsening clinical scenario there was a question about whether or not the patient was still shedding virus for prognostic and therapeutic implications. Therefore, his SARS-CoV-2 test by polymerase chain reaction nasopharyngeal was positive again on day 18 . On day 20 , the patient developed leukocytosis, his fever persisted, and a portable chest radiograph revealed extensive bilateral pulmonary opacities with focal worsening in left lower base. Due to this constellation of findings, a vancomycin IV (1,500 mg once) was started for empirical treatment of hospitalacquired pneumonia. Sputum samples obtained on day 20 revealed Staphylococcus aureus on subsequent days.

From a hematologic perspective, on day 9 due to challenges to maintain a therapeutic level of anticoagulation with heparin infusion thought to be related to antithrombin deficiency, anticoagulation was changed to argatroban infusion $(0.5 \mathrm{mcg} / \mathrm{kg} / \mathrm{min}$ targeting a PTT of 70-105 seconds) for ongoing management of CAC. Although D-dimer was $>20 \mathrm{mcg} / \mathrm{mL}$ FEU on admission and on days 4 and 5, D-dimer trended down to $12.5 \mathrm{mcg} / \mathrm{mL}$ FEU on day 16 .

Throughout the patient's hospital stay, no significant bleeding was seen. Hemoglobin was $15.2 \mathrm{~g} / \mathrm{dL}$ on admission, but anemia developed with a nadir of $6.5 \mathrm{~g} / \mathrm{dL}$, warranting transfusion of red blood cells on day 22. Platelet count was 165,000 per microliter on admission and remained within normal limits until platelet clumping was noted on day 15 laboratory collection.

Hematology was consulted on day 20 to obtain an accurate platelet count. A peripheral blood smear from a sodium citrate containing tube was remarkable for prominent platelet clumping, particularly at the periphery of the slide (Figure 1). Platelet clumping was reproduced in samples containing EDTA and heparin. Other features of the peripheral blood smear included the presence of echinocytes with rare schistocytes. To investigate for presence of disseminated intravascular coagulation on 
day 22, fibrinogen was found to be mildly elevated at $538 \mathrm{mg} / \mathrm{dL}$ (normal range: $243-517 \mathrm{mg} / \mathrm{dL}$ ) and a D-dimer value of $11.96 \mathrm{mcg} / \mathrm{mL}$ FEU.

On day 22, the patient's ventilator requirements escalated to requiring $100 \%$ $\mathrm{FiO}_{2}$ and $10 \mathrm{~cm} \mathrm{H}_{2} \mathrm{O}$ of positive endexpiratory pressure with mean arterial pressures in the 50 to $60 \mathrm{~mm} \mathrm{Hg}$ range. Within 30 minutes an electrocardiogram (EKG) obtained revealed a STEMI (Figure 2). Troponin was measured at $0.65 \mathrm{ng} / \mathrm{mL}$ (normal range: $0.02-0.06 \mathrm{ng} / \mathrm{mL}$ ). Just after an EKG was performed, the patient developed a ventricular fibrillation arrest and was unable to obtain return of spontaneous circulation. The patient was pronounced dead. The family declined an autopsy.

\section{DISCUSSION}

Pseudothrombocytopenia, or platelet clumping (agglutination), is estimated to be present in up to $2 \%$ of hospitalized patients. ${ }^{4}$ Pseudothrombocytopenia was found to be the root cause of thrombocytopenia hematology consultations in up to $4 \%$ of hospitalized patients. ${ }^{5}$ The etiology is commonly ascribed to EDTA inducing a conformational change in the GpIIb-IIIa platelet complex, rendering it susceptible to binding of autoantibodies, which cause subsequent platelet agglutination. ${ }^{6}$ In most cases (83\%), the use of a non-EDTA anticoagulant, such as sodium citrate, resolves the platelet agglutination and allows for accurate platelet count reporting. ${ }^{4}$ Pseudothrombocytopenia in most cases is considered an in vitro finding without clinical relevance. ${ }^{7}$ However, in this patient's case, his pan-pseudothrombocytopenia was temporally associated with an arterial occlusive event (STEMI) leading to his demise despite therapeutic anticoagulation in the setting of CAC. This temporal association raises the possibility that pseudothrombocytopenia seen on the peripheral blood smear is an accurate representation of in vivo activity.

Pseudothrombocytopenia has been associated with sepsis from bacterial and viral causes as well as autoimmune and medication effect. ${ }^{4,8-10} \mathrm{Li}$ and colleagues reported transient EDTA-dependent pseudothrombocytopenia in a patient with COVID-19 infection; however, platelet clumping resolved with use of a citrate tube, and the EDTA- dependent pseudothrombocytopenia phenomenon resolved with patient recovery. ${ }^{11}$ The frequency of COVID-19-related pseudothrombocytopenia is currently unknown.

Although the understanding of COVID19-associated CAC continues to evolve, it seems that initial reports support the idea that hemostatic dysfunction tends to more thrombosis than to bleeding. ${ }^{12}$ Rather than overt disseminated intravascular coagulation with reduced fibrinogen and bleeding, CAC is more closely associated with blood clotting, as demonstrated by autopsy studies revealing microvascular thrombosis in the lungs. ${ }^{13}$ The D-dimer test has been identified as the most useful biomarker by the International Society of Thrombosis and Hemostasis to screen for CAC and stratify patients who warrant admission or closer monitoring. ${ }^{12}$ Other identified features of CAC include prolonged prothrombin time and thrombocytopenia. ${ }^{12}$

There have been varying clinical approaches to CAC management. A retrospective review found that prophylactic heparin doses were associated with improved mortality in those with elevated D-dimer $>3.0 \mathrm{mg} / \mathrm{L} .{ }^{14}$ There continues to be a diversity of varying clinical approaches with many medical centers advocating for an intensified prophylactic twice daily low molecular-weight heparin compared with others advocating for full therapeutic dose anticoagulation for patients with elevated D-dimer. ${ }^{15}$ This patient was treated aggressively with full-dose anticoagulation, and despite his having a down-trend in D-dimer, he suffered a lethal arterial thrombosis in the form of a STEMI.

Varatharajah and Rajah believe that CAC is more closely aligned with endotheliopathyassociated vascular microthrombotic disease (EA-VMTD) ${ }^{16}$ EA-VMTD involves a disequilibrium state between insufficient ADAMTS13 enzyme and excessive exocytosis of ultralarge von Willebrand factor (ULvWF) multimers from endothelial cells affected by COVID-19. This theory endorses that ULvWF multimers cause platelet adhesion and subsequent rapid platelet activation, causing platelet aggregation and formation of microthrombi. ${ }^{17}$ As these platelet aggregates grow to a certain point, they can no longer remain adhered to ULvWF, causing these platelet aggregates to be released 
into the circulation and causing thrombotic sequelae. ${ }^{16}$ Therefore, a plausible explanation for the patient's pan-pseudothrombocytopenia may be the detection of these circulating platelet aggregates, which, in turn, was the same process leading to his STEMI. Interestingly, this patient's fatal arterial event occurred in the presence of therapeutic anticoagulation, raising the question of whether other therapeutic interventions to treat CAC, such as further antithrombotic therapy (eg, aspirin, clopidogrel) or novel strategies would be of benefit.

\section{CONCLUSIONS}

This patient's case highlights the presence of pan-pseudothrombocytopenia despite the use of a sodium citrate and heparin containing tube in a COVID-19 infection with multiorgan dysfunction. This developed 1 week prior to the patient suffering a STEMI despite therapeutic anticoagulation. Although the exact nature of CAC remains to be worked out, it is possible that platelet agglutination/clumping seen on the peripheral blood smear is representative of in vivo activity and serves as a harbinger for worsening thrombosis. The frequency of such phenomenon and efficacy of further interventions has yet to be explored.

\section{Author disclosures}

The authors report no actual or potential conflicts of interest with regard to this article.

\section{Disclaimer}

The opinions expressed herein are those of the authors and do not necessarily reflect those of Federal Practitioner, Frontline Medical Communications Inc., the US Government, or any of its agencies. This article may discuss unlabeled or investigational use of certain drugs. Please review the complete prescribing information for specific drugs or drug combinations -including indications, contraindications, warnings, and adverse effects-before administering pharmacologic therapy to patients.

\section{References}

1. World Health Organization. Naming the coronavirus disease (COVID-19) and the virus that causes it. https://www .who.int/emergencies/diseases/novel-coronavirus-2019 /technical-guidance/naming-the-coronavirus-disease -(COVID-2019)-and-the-virus-that-causes-it. Accessed July $15,2020$.

2. Ghelichkhani P, Esmaeili M. Prone position in management of COVID-19 patients; a commentary. Arch Acad Emerg Med. 2020;8(1):e48. Published 2020 April 11.
3. National Library of Medicine, Clinicaltrials.gov. Expanded access to convalescent plasma for the treatment of patients with COVID-19. NCT04338360. https://clinicaltri als.gov/ct2/show/nct04338360. Update April 20, 2020. Accessed July 15, 2020.

4. Tan GC, Stalling M, Dennis G, Nunez M, Kahwash SB. Pseudothrombocytopenia due to platelet clumping: a case report and brief review of the literature. Case Rep Hematol. 2016;2016:3036476. doi:10.1155/2016/3036476

5. Boxer M, Biuso TJ. Etiologies of thrombocytopenia in the community hospital: the experience of 1 hematologist. Am J Med. 2020;133(5):e183-e186. doi:10.1016/j.amjmed.2019.10.027

6. Fiorin F, Steffan A, Pradella P, Bizzaro N, Potenza R, De Angelis V. IgG platelet antibodies in EDTA-dependent pseudothrombocytopenia bind to platelet membrane glycoprotein IIb. Am J Clin Pathol. 1998;110(2):178-183. doi:10.1093/ajcp/110.2.178

7. Nagler M, Keller P, Siegrist S, Alberio L. A case of EDTADependent pseudothrombocytopenia: simple recognition of an underdiagnosed and misleading phenomenon. BMC Clin Pathol. 2014;14:19. doi:10.1186/1472-6890-14-19

8. Mori M, Kudo H, Yoshitake S, Ito K, Shinguu C, Noguchi T. Transient EDTA-dependent pseudothrombocytopenia in a patient with sepsis. Intensive Care Med. 2000;26(2):218-220. doi:10.1007/s001340050050.

9. Choe W-H, Cho Y-U, Chae J-D, Kim S-H. 2013. Pseudothrombocytopenia or platelet clumping as a possible cause of low platelet count in patients with viral infection: a case series from single institution focusing on hepatitis A virus infection. Int J Lab Hematol. 2013;35(1):70-76. doi:10.1111/j.1751-553x.2012.01466.

10. Hsieh AT, Chao TY, Chen YC. Pseudothrombocytopenia associated with infectious mononucleosis. Arch Pathol Lab Med. 2003;127(1):e17-e18. doi:10.1043/0003-9985(2003)1272.0.CO;2

11. Li H, Wang B, Ning L, Luo Y, Xiang S. Transient appearance of EDTA dependent pseudothrombocytopenia in a patient with 2019 novel coronavirus pneumonia [published online ahead of print, 2020 May 5]. Platelets. 2020;1-2. doi:10.1080/09537104.2020.1760231

12. Thachil J, Tang N, Gando S, et al. ISTH interim guidance on recognition and management of coagulopathy in COVID-19. J Thromb Haemost. 2020;18(5):1023-1026. doi:10.1111/jth.14810

13. Magro C, Mulvey $\mathrm{JJ}$, Berlin D, et al. Complement associated microvascular injury and thrombosis in the pathogenesis of severe COVID-19 infection: a report of five cases. Trans/ Res. 2020;220:1-13. doi:10.1016/j.trsl.2020.04.007

14. Tang N, Bai H, Chen X, Gong J, Li D, Sun Z. Anticoagulant treatment is associated with decreased mortality in severe coronavirus disease 2019 patients with coagulopathy. J Thromb Haemost. 2020;18(5):1094-1099. doi:10.1111/jth.14817

15. Connors JM, Levy JH. COVID-19 and its implications for thrombosis and anticoagulation. Blood. 2020;125(23):20332040. doi.org/10.1182/blood.2020006000.

16. Varatharajah N, Rajah S. Microthrombotic complications of COVID-19 are likely due to embolism of circulating endothelial derived ultralarge von Willebrand factor (eULVWF) Decorated-Platelet Strings. Fed Pract. 2020;37(6):258-259. doi:10.12788/fp.0001

17. Bernardo A, Ball C, Nolasco L, Choi H, Moake JL, Dong JF. Platelets adhered to endothelial cell-bound ultra-large von Willebrand factor strings support leukocyte tethering and rolling under high shear stress. J Thromb Haemost. 2005;3(3):562-570. doi:10.1111/j.1538-7836.2005.01122.x 